\title{
Improved Performance of CLOUDQUAL in Internet of Things
}

\author{
S. Saravanan ${ }^{1}$, S. Sowmiya ${ }^{2}$ \\ Assistant Professor, Department of CSE, M Kumarasamy College of Engineering, Karur, Tamil Nadu, India ${ }^{1,2}$
}

\begin{abstract}
Distributed computing is an essential component of the foundation of the Internet of Things (IoT). Mists will be expected to bolster enormous quantities of communications with variable quality necessities. Benefit quality can accordingly be an imperative soul among cloud providers. in order to distinguish themselves from their rivals, cloud provider looked to give predominant administrations that live up to clients' desires. a top quality model are regularly acclimated speak to, measure, and think about the standard of the providers, such a shared fondness are frequently settled among cloud partners. amid this paper, we tend to take an administration point of view and start a top quality model named CLOUDQUAL for cloud administrations. it's a model with quality measurements and measurements that objectives general cloud administrations. CLOUDQUAL contains six quality dimensions, i.e., ease of use, accommodation, dependableness, responsiveness, security, and snap, of that convenience is subjective.
\end{abstract}

Keywords: Internet of Things, CLOUDQUAL, usability, convenience, dependableness.

\section{INTRODUCTION}

Cloud benefits square measure connected in Internet-based situations. Thus, they impart couple of similitudes to customary administrations that square measure conveyed in human-based situations. a fantastic model of cloud administrations indicates quality measurements and measurements to detail and evaluate benefit quality. It makes general learning of administration quality, i.e., what it implies and the best approach to live it, such once a fantastic measurement like dependability is said, it proposes that exactly a similar issue to two gatherings and furthermore a similar metric is received to live it. With a quality model, cloud clients will ensure regardless of whether administrations are given the normal quality, and may kill potential distortion. Thus, an excellent model is in a position to shield cloud customers' interests.

\section{II.RELATED WORKS}

It's effortful to build up trust between a couple of gatherings that is essential to leading business in Internetbased situations. In the event that cloud providers still create tinier no dedication, as they're doing right now, it definitely hurts their businesses [1]. As cloud customers mind identifying with nature of service $(\mathrm{QoS})$, people that don't receive an astounding model and make no dedication in today's market may not survive [2]. Surely, without quality model, cloud providers can't guarantee themselves that their genuinely unrivaled administrations are not unacknowledged [3][6]. This paper present the vital thoughts of transmission distributed computing and displays a totally one of a kind system. Address transmission distributed computing from transmissionmindful cloud (media cloud) and cloud-mindful mixed media (cloud media) views.[2] Media Cloud is AN open supply stage that licenses scientists to answer quantitative inquiries on the substance of on-line media. abuse Media Cloud, analysts, reporting pundits and intrigued voters will analyze what media sources cowl that stories[1]

\section{PROPOSED WORKS}

CLOUDQUAL may well be a top quality model that we have a twisted to propose for cloud Services a top quality model edges cloud providers as well and makes getable a curiously large scaled probability of cloud administrations. The paper's primary commitments square measure as follows. 1) A prime quality model for cloud administrations, said as CLOUDQUAL, which determines six quality measurements and five quality measurements: it is a model with quality measurements and measurements that objectives general cloud services. 2) A contextual analysis including three certifiable stockpiling mists: Our trial comes about demonstrate that CLOUDQUAL can assess their quality that exhibits its effectiveness [4].

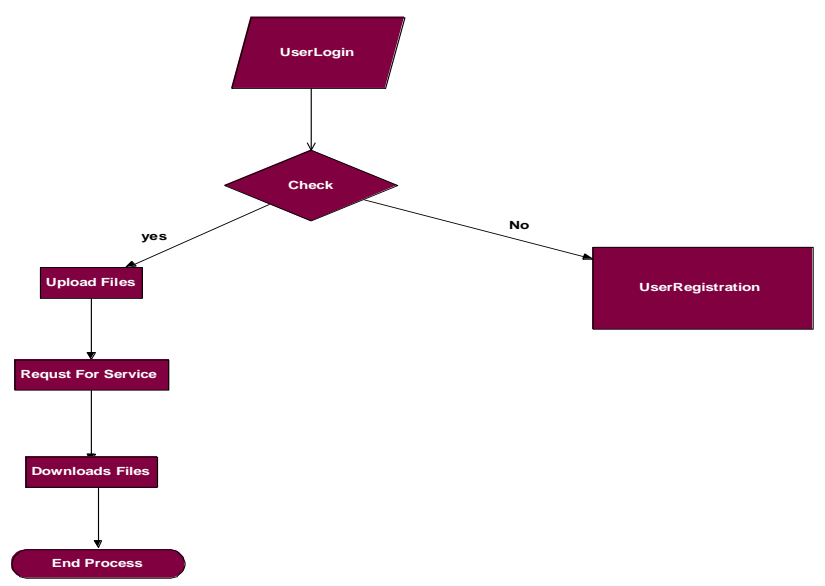

Fig 1 User 
3) A technique to formally approve a prime quality model wherever the cloud storage are often created secure. Figure using standard criteria, to be specific, connection, three server application is run to examine if it retrieves the consistency, and discriminative power: we have a bowed required data from remote machines and thereby the to show that CLOUDQUAL will separate administration applying is tested to examine for consistency of output and quality model for cloud administrations should be for varied styles of computer file.

objective, calculable, and evident, so as that cloud providers can gage the QoS conveyed, and cloud customers can approve the QoS.

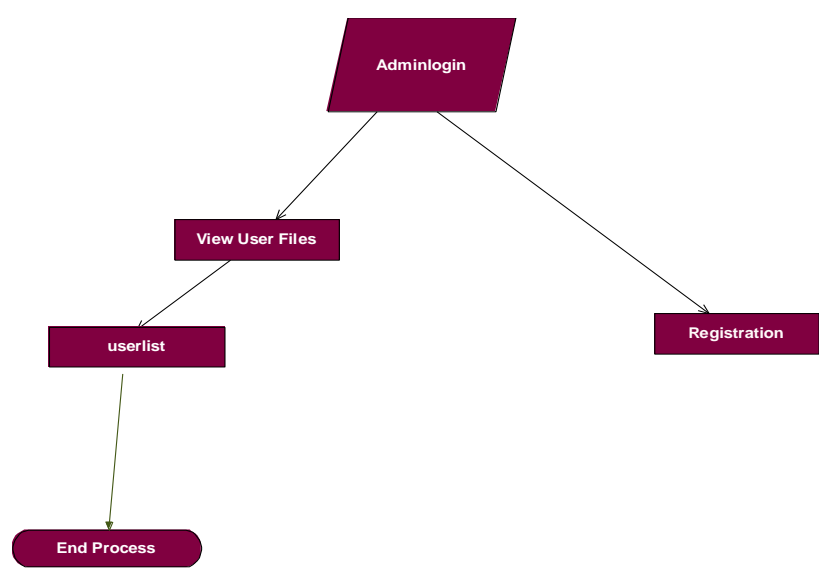

Fig 2: Admin

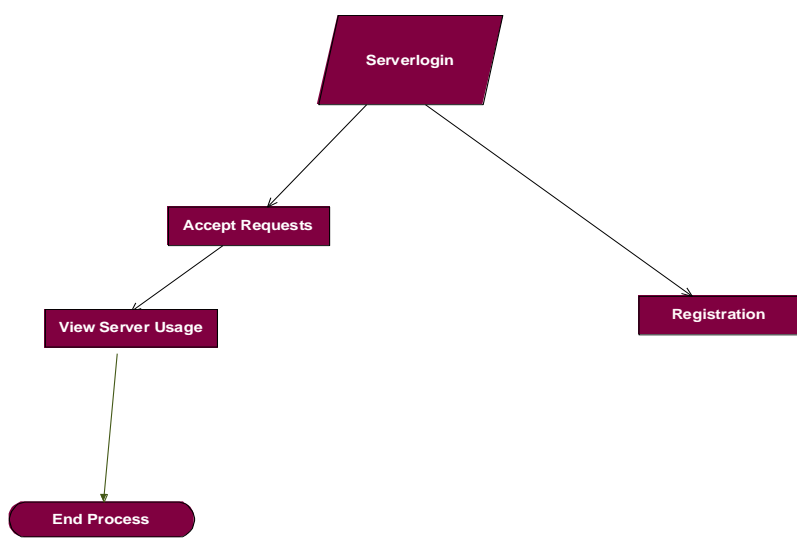

Fig 3: Server

\section{SYSTEM MODEL}

User store great deal knowledge of clouds and access data victimization secure key provided admin when encrypting data's. encode the info victimization SECY. User store information when auditor, read and supportive information and conjointly modified information. User once more views information at that point admin provided the message to user solely changes information. Figure two during this module admin, read cluster request from cluster owner and conjointly settle for the cluster request[7]. Conjointly read user details and cluster user details. Figure one during this module, Users square measure having authentication and security to access the detail that is bestowed within the metaphysics system. Before accessing or looking the main points user ought to have the account therein otherwise they ought to register initial user will register their details like name, password, gender, age, and then. We tend to develop this module,

\section{RESULT COMPARISONS}

The module regarding the remote access of the server is additionally enforced in a web put in setting. The complete desktop management of any server of any network is retrieved sanctioning to manage the complete network within the net. The software system is enforced by giving the IP Address of the server foreign countries and located to figure as supposed. The grid resolution facility additionally the compression level also operating in a very good manner. It is thus suggested to attach the server within the net by scientific discipline Address and so management the network that permits the desktop management of the hosts conjointly. The server will be connected in any system supplied with a web server. User validation is that stage of the project once the theoretical style is becomes a operating system. When testing the modules with success, the required privileges ar given to the users. All the users ar requested to handle the system fastidiously. The important time issues that occur are with success solved. The target is to place the tested system into operation. It consists of testing the developed program with sample information. Detection and corrections of errors. creating necessary changes within the system. Checking of reports. making pc compatible files. Web Services (AWS) programming framework Developer's Kit (SDK) for Java gives a Java API to Amazon S3. With it, designers will begin in minutes with one, downloadable bundle that components the AWS Java library, code tests, and documentation. Amazon S3 conjointly offers a WUI for web clients. With it, userscan just perform deliver, Read, Update, and Delete (CRUD)operations on AmazonS3.Upload, download, and erase operations ar analyzed underneath suppose responsiveness. Expect here that the most extreme adequate time - a predefined parameter - to finish each operation is five hundred $\mathrm{s}$ on a ten MB document $-a$ reasonable range we have a tendency to see in our analyses. To begin with, we have a tendency to play out AN exchange operation on Amazon S3. inside the exploratory sum, we tend to exchange compressed record of ten $\mathrm{MB}$ from our desktop to Amazon S3. It takes $38.940 \mathrm{~s}$ on the normal with a common deviation of three.565 to complete the operation. In our examinations, Aliyun OSS spends longer to complete the exchange operation than Amazon S3. It takes $318.862 \mathrm{~s}$ by and large with a regular deviation of thirtynine. 371 to complete the operation, that is A request of extent slower than that of Amazon S3. Utilizing (3), Aliyun OSS's end-to-end responsiveness is computed as zero. 362 .

We conjointly play out an exchange operation and an erase operation on the ten MB document on Amazon S3, Azure 
Blob, and Aliyun OSS. We summarize the prompts to Table II. look for counsel from for the little print. Fig. a couple of demonstrates the end-to-end responsiveness came to in finishing the exchange, download, and erase operations for the three stockpiling mists. So as to create a whole picture of responsiveness, we have a tendency to fluctuate the extent of documents to be one and one hundred MB, and play out the exchange, download, and erase operations on Amazon S3, Azure Blob, ANdAliyun OSS too once the size of records will increment by a request of size, the time that the 3 stockpiling mists pay increments by indistinguishable request for the exchange operation and in this way the exchange and timeframe ar thirty one days and zero days, severally. Along these lines, its end to-end accessibility is also one hundred.

\section{CLOUD QUALITY (CLOUDQUAL)}

We particularly need to work out regardless of whether our quality model will separate administration quality, as far as the standard measurements and measurements per CLOUDQUAL. The specialized typical that we receive to approve CLOUDQUAL is IEEE STD 1061-1998(R2009) (i.e., IEEE ordinary for a product framework Quality Metrics Methodology). the quality gives "a philosophy for setting up quality needs and recognizing, executing, breaking down, and affirming strategy and stock programming framework quality measurements". It "applies to all or any product framework in any regard periods of any product life cycle," and goes for "measuring or surveying the nature of programming framework." particularly, a metric should have a high level of relationship with the standard measurement that it speaks to be pondered legitimate. Subsequently, we tend to ar required with 3 legitimacy criteria for every the quality, i.e., relationship, consistency, and discriminative power.

\section{VALIDATE CLOUDQUAL}

Relationship: Let be the straight parametric measurement between an astounding measurement and a metric. The relationship measure needs that the variety inside the quality measurement values clarified by the variety inside the metric qualities, i.e., ,ought to surpass a predefined edge . Truth be told, this model surveys regardless of whether an adequately solid direct affiliation exists between a brilliant measurement and a metric, determined the metric will be utilized as A pointer for the standard measurement.

Consistency: Let be the rank parametric measurement of matched estimations of a top notch measurement and a metric, wherever a great measurement and a metric attempt is measured for indistinguishable administration. The consistency measure needs that totally the value of should surpass a predefined edge . Truth be told, this basis surveys regardless of whether a metric will precisely rank a gathering of administrations by an amazing measurement.
Discriminative Power: Let be a certainty level. Utilize possibility table to convey quality measurement and metric values and figure the chi-square ( ) datum. The discriminative power measure needs that this value should surpass the chisquarestatistic cherish. Truth be told, this model surveys whether a metric will isolate a gathering of superb administrations from resource of low-quality ones for a great measurement. In option words, the arrangement of metric estimations of the past should be altogether not the same as those of the last mentioned. Download and erase responsiveness will be substantial in a comparable way. For the clarification of conciseness, we have a tendency to preclude those parts. look for counsel from for the approval ofAVAL, REL, SECY, andELAS.

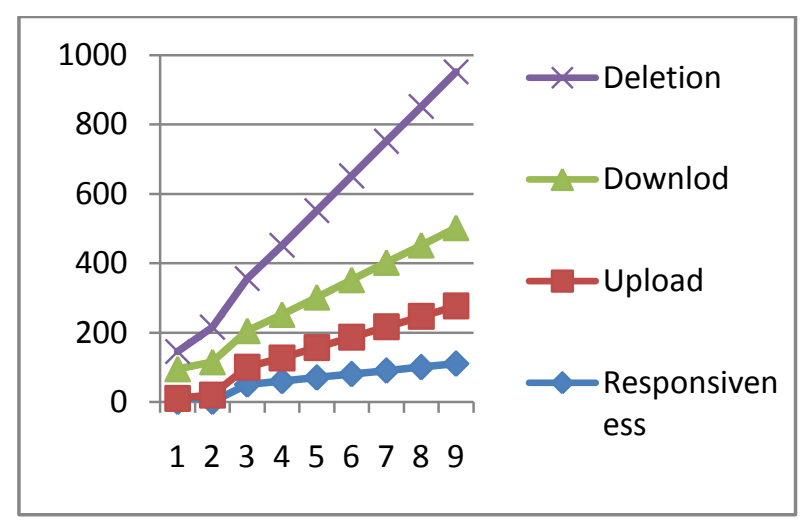

Fig 4 Performance of CLOUDQUAL

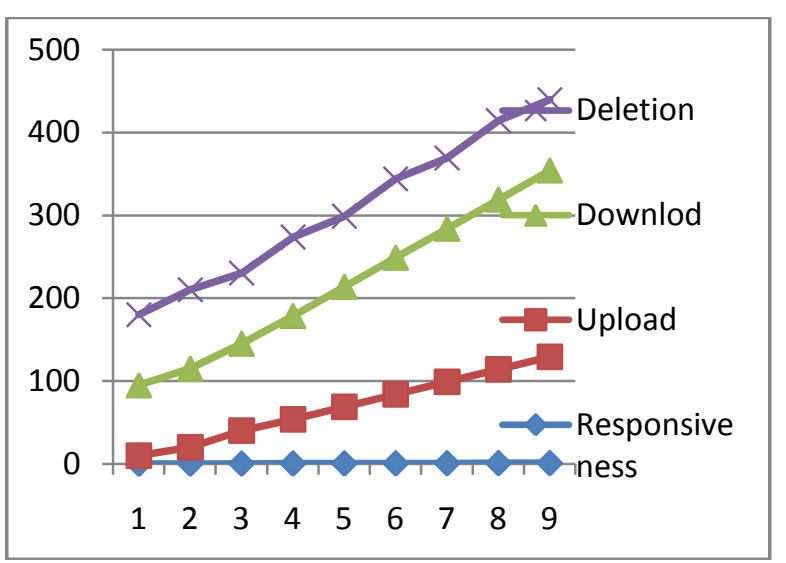

Fig 5 Comparative of Cloud qulaity

\section{CONCLUSION}

Distributed computing is an essential part of the foundation of the IoT. Mists will be expected to bolster mammoth quantities of connections with differed quality necessities. Benefit quality can along these lines be an indispensable individual among cloud providers. we have a tendency to exhibit inside the adequacy of CLOUDQUAL, lead experimental contextual analyses on 2 stockpiling mists, i.e., claim destinations. Comes about demonstrate that CLOUDQUAL will esteem their quality. To exhibit the soundness of CLOUDQUAL, we have a tendency to approve it with 3 criteria,i.e., connection, consistency, and discriminative power. 


\section{REFERENCES}

[1] Thilagamani, S. and N. Shanthi, 2010. Literature survey on enhancing cluster quality. Int. J. Comput. Sci. Eng.., 2: 1999-2002. http://www.enggjournals.com/ijcse/doc/IJCSE10- 02-06-26.pdf

[2] S. Chitra, B. Madhusudhanan, G. Sakthidharan, P. Saravanan, Local Minima Jump PSO for Workflow Scheduling in Cloud Computing Environments, Springer, ISBN 364241673X, 12251234, 2014.

[3] E.T. Venkatesh , P. Thangaraj , and S. Chitra , “ An Improved Neural Approach for Malignant and Normal Colon Tissue Classification from Oligonucleotide Arrays ," European J. Scientific Research, vol. 54, pp. 159-164, 2011.

[4] Iosup et al.,"Performance Analysis of Cloud Computing Services for Many-Tasks Scientific Computing," IEEE Trans. Parallel and Distributed Systems, Vol. 22, no. 6, pp. 931-45,2011.

[5] Javadi, D. Kondo, J. M. Vincent, and D. P. Anderson,"Discovering statistical models of availability in large distributed systems An empirical study of SETI@home," IEEE Trans. Parallel Distrib. Syst., Vol.22, no. 11, pp. 1045-9219,2011.

[6] S. Ferretti et al., "QoS-aware clouds," in Proc. 3rd Int. Conf. Cloud Comput.(CLOUD), Miami, FL, USA, 2010, pp. 321328.

[7] S. K. Garg, S. Versteeg, and R. Buyya, "A framework for ranking of cloudcomputing services," Future Gener. Comput. Syst., vol. 29, no. 4,pp. 1012-1023, 2013.

[8] S. Li, L. Xu, and X. Wang, "Compressed sensing signal and data acquisitionin wireless sensor networks and Internet of things," IEEE Trans. Ind.Informat., vol. 9, no. 4, pp. 2177-2186, Nov. 2013. 